PAPER

\title{
Stroke motor recovery: active neuromuscular stimulation and repetitive practice schedules
}

\author{
J H Cauraugh, S B Kim
}

See Editorial Commentary p 1465

J Neurol Neurosurg Psychiatry 2003;74:1562-1566

See end of article for authors' affiliations ......................

Correspondence to: Dr James Cauraugh, Motor Behavior Laboratory, PO Box 118206, University of Florida, Gainesville, Florida 32611 , USÁ; icaura@hhp.ufl.edu

Received 12 February 2003 In revised form

9 April 2003

Accepted 10 May 2003
Objective: To investigate progress toward motor recovery in patients with chronic hemiparesis (mean time since stroke 3.2 years), comparing different types of practice schedules.

Design: To increase voluntary control of the upper extremity, active neuromuscular stimulation was administered during blocked and random practice schedules as patients performed three specific movements: wrist/finger extension, elbow joint extension, and shoulder joint abduction.

Methods: 34 stroke subjects volunteered to participate and were randomly assigned to one of three treatment groups: blocked practice (the same movement was repetitively performed on successive trials) combined with active neuromuscular stimulation; random practice (different movements on successive trials) along with active stimulation; or no active stimulation assistance control group. Subjects completed two days of 90 minute training for each of two weeks with at least 24 hours of rest between sessions. A session was three sets of 30 successful active neuromuscular stimulation trials with the three movements executed 10 times/set.

Results: Mixed design analyses on three categories of behavioural measures indicated motor improvements for the blocked and random practice/stimulation groups in comparison with the control group during the post-test period, with a larger number of blocks moved, faster premotor and motor reaction times, and less variability in the sustained muscular contraction task.

Conclusions: Upper extremity rehabilitation intervention of active stimulation and blocked practice performed as well as stimulation/random practice. Moreover, these purposeful voluntary movement findings support and extend sensorimotor integration theory to both practice schedules.
C hronic stroke patients with partial paralysis must relearn purposeful voluntary movement control. $^{1-4}$ Movement practice is often administered in a blocked schedule-the same movement is repetitively attempted on successive trials. However, repetitively executing the same movement consecutively may not be the most efficient way of relearning motor actions. A strong line of motor learning research challenges the notion that blocked practice is the best way to improve learning..$^{5-7}$

The type of repetitive practice that individuals go through while learning motor skills presents a contradiction known as contextual interference-functional interference in practice situations because of the presence of multiple movements. ${ }^{7}$ Early in learning, blocked practice excels in comparison to random practice. On the other hand, later in learning, random practice is superior to blocked. ${ }^{78}$

Contextual interference has not been empirically investigated in stroke rehabilitation. Perhaps one of the reasons is the difficulty in voluntarily activating purposeful movements. However, there is convincing evidence in recent studies supporting motor improvements from residual hemiparesis when voluntary movements are supplemented by active neuromuscular stimulation. ${ }^{19-13}$ The theoretical basis for these motor improvements is sensorimotor integration theory, in that the somatosensory cortex interacts extensively with the motor cortex during motor learning. ${ }^{3}{ }^{14} 15$ Indeed, neural mechanisms in the cerebral cortex association areas integrate sensory and motor functions during the perception/ action of executing voluntary purposeful movement. ${ }^{14}$

The critical question is, what is the most efficient and effective practice schedule to improve motor recovery after at least one year after a stroke? Bernstein argued that practice, when properly undertaken, does not consist of merely repeating the same motor solution trial after trial, but in the process of solving the motor problem repeatedly with techniques changed and refined across repetitions. ${ }^{16}$ Thus blocked practice is limited because only one solution to the motor problem is continuously repeated. In contrast, random practice necessitates different movement solutions on successive trials.

However, no studies have directly compared practice schedules during upper extremity motor recovery progress. Thus, the primary purpose was to investigate blocked versus random practice on motor recovery of three different upper extremity movements assisted by active neuromuscular stimulation. A second purpose focused on sensorimotor integration theory; both blocked and random practice groups were hypothesized to demonstrate motor recovery progress from chronic hemiparesis.

\section{METHODS}

\section{Subjects}

Specific characteristics of each subject are listed in table 1. Subjects read and signed an institutional review board/ethics committee approved informed consent before being tested. Admission criteria were:

- diagnosis of no more than two strokes;

- a lower limit of $10^{\circ}$ of voluntary wrist/finger extension from a $90^{\circ}$ wrist flexed position;

- an $80 \%$ upper limit of motor recovery as assessed by rectified electromyographic (EMG) activation patterns and force generation while directly comparing the impaired and unimpaired limbs ${ }^{17}{ }^{18}$;

- absence of other neurological deficits;

- currently not participating in another upper extremity rehabilitation protocol. 


\begin{tabular}{|c|c|c|c|c|c|}
\hline $\begin{array}{l}\text { Practice group/ } \\
\text { subject No }\end{array}$ & Sex & Age (years) & $\begin{array}{l}\text { Post stroke time } \\
\text { (years) }\end{array}$ & CVA location & Number of CVAs \\
\hline Blocked 1 & Male & 68.8 & 3.6 & Right hem & 1 \\
\hline Blocked 2 & Male & 81.2 & 8.1 & Left hem & 2 \\
\hline Blocked 3 & Male & 57.1 & 2.6 & Left hem & 1 \\
\hline Blocked 4 & Male & 63.5 & 2.8 & Left hem & 1 \\
\hline Blocked 5 & Male & 76.2 & 3.9 & Right hem & 1 \\
\hline Blocked 6 & Male & 51.1 & 1.8 & Right hem & 1 \\
\hline Blocked 7 & Male & 48.6 & 4.0 & Left hem & 2 \\
\hline Blocked 8 & Female & 69.4 & 1.1 & Right hem & 1 \\
\hline Blocked 9 & Female & 53.7 & 1.4 & Left hem & 1 \\
\hline Blocked 10 & Male & 69.8 & 1.2 & Left hem & 2 \\
\hline Blocked 11 & Male & 69.3 & 3.8 & Right hem & 1 \\
\hline Blocked 12 & Male & 63.3 & 2.6 & Left hem & 1 \\
\hline Blocked 13 & Male & 57.4 & 2.8 & Left hem & 1 \\
\hline Blocked 14 & Male & 82.7 & 4.8 & Right hem & 1 \\
\hline Random 1 & Male & 66.7 & 5.3 & Left hem & 1 \\
\hline Random 2 & Male & 77.9 & 3.7 & Right hem & 1 \\
\hline Random 3 & Male & 68.1 & 2.8 & Right hem & 2 \\
\hline Random 4 & Male & 81.1 & 8.0 & Left hem & 1 \\
\hline Random 5 & Male & 77.3 & 3.8 & Right hem & 1 \\
\hline Random 6 & Male & 74.4 & 2.4 & Right hem & 1 \\
\hline Random 7 & Male & 65.1 & 3.0 & Left hem & 1 \\
\hline Random 8 & Male & 63.3 & 2.7 & Left hem & 1 \\
\hline Random 9 & Male & 58.3 & 2.9 & Left hem & 1 \\
\hline Random 10 & Male & 70.7 & 1.5 & Right hem & 2 \\
\hline Random 11 & Male & 63.4 & 2.8 & Left hem & 1 \\
\hline Random 12 & Female & 51.7 & 2.3 & Left hem & 1 \\
\hline Random 13 & Male & 54.1 & 1.6 & Right hem & 1 \\
\hline Random 14 & Male & 69.8 & 3.3 & Right hem & 2 \\
\hline Control 1 & Male & 69.2 & 1.2 & Left hem & 2 \\
\hline Control 2 & Female & 51.9 & 1.2 & Left hem & 1 \\
\hline Control 3 & Male & 81.0 & 1.3 & Right hem & 1 \\
\hline Control 4 & Male & 59.7 & 7.3 & Left hem & 2 \\
\hline Control 5 & Male & 63.6 & 2.9 & Left hem & 1 \\
\hline Control 6 & Male & $\begin{array}{l}69.5 \\
\mathrm{~m}=66.1\end{array}$ & $\begin{array}{l}4.3 \\
m=3.2\end{array}$ & Right hem & 1 \\
\hline
\end{tabular}

\section{Measurement instruments and procedure}

Motor functions of the upper extremity were evaluated with three categories of measurement. Subjects completed a functional manual dexterity box and block test. ${ }^{19}$ For 60 seconds, participants repetitively attempted to grasp a $2.54 \mathrm{~cm}$ block, transport it over a $14.3 \mathrm{~cm}$ barrier, release it on the other side, and return to the original side for another block.

Chronometric reaction time and sustained muscle contraction tasks were included to determine the ability to modulate purposeful muscle activation and force production. ${ }^{12021}$ These measures were collected while subjects completed isometric wrist/finger extension movements against $34.09 \mathrm{~kg}$ load cells, and muscle activity was recorded by surface electrodes. For the reaction time task, subjects reacted to the onset of an auditory stimulus by lifting the wrist/fingers against the cushioned platforms/load cell(s). Ten trials were administered to the impaired and unimpaired limbs separately (unilateral testing) and to both limbs simultaneously (bilateral testing). In the sustained task, an auditory stimulus signalled first, a deliberate increase in the force generated against the load cell(s), and second, maintenance of that maximum level for at least five seconds. Three trials were completed for each hand separately and together. These tasks were counterbalanced across subjects, and no stimulation was provided during either task.

\section{Practice schedules and active stimulation: training procedure}

Stroke patients were randomly assigned to one of three motor recovery practice groups with two restrictions: (1) 28 patients were assigned to the blocked and random practice groups, and (2) six patients were assigned to the control group. As recommended by Altman and Schulz, a group assignment randomisation schedule was created before testing began to conceal treatment allocation. ${ }^{22}$ Group assignment was checked on the master list and subjects were informed after qualified patients successfully completed the pretest.

For the active neuromuscular stimulation assistance, surface electrodes were attached to three sets of primary muscles in the impaired upper extremity for the practice schedule groups:

- extensor communis digitorum and extensor carpi ulnaris: wrist/finger extension;

- triceps brachii: elbow extension;

- anterior and middle deltoid: shoulder joint abduction.

For each movement, subjects voluntarily generated a target threshold level of EMG activity. As soon as the threshold was met, an Automove EMG facilitator microprocessor immediately provided an electrical stimulation that assisted the muscles through a full range of motion. The settings for the electrical stimulation were one second ramp up, five seconds of biphasic stimulation at $50 \mathrm{~Hz}$ (mA range 13 to 28 , pulse width of $200 \mu \mathrm{s}$ ), and one second ramp down. The initial threshold was set at $50 \mu \mathrm{V}$. When subjects achieved the threshold level, the microprocessor automatically increased the target level higher for the next trial. If the target level was not met, then the microprocessor lowered the threshold level. Consecutive trials were separated by 25 seconds of rest as subjects relaxed their muscles.

For the blocked practice group, 10 consecutive movement trials were executed before the surface electrodes were 


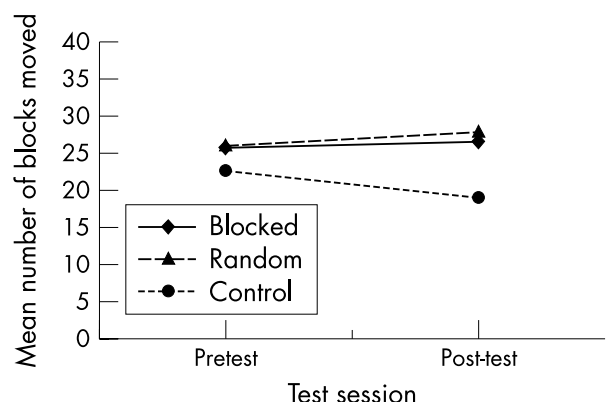

Figure 1 Mean number of blocks moved for the motor recovery practice schedules as a function of test sessions.

disconnected from the microprocessor and replaced with electrodes for another set of muscles/movement. However, in the random practice group, electrodes for different muscles/ movements were changed after each trial.

Subjects completed two days of 90 minute training for each of two weeks with at least 24 hours of rest between sessions. A session was three sets of 30 successful active neuromuscular stimulation trials with the three movements executed 10 times per set for blocked and random practice. The control group, however, did not receive any active stimulation for the three movements. For 90 minutes/day over four days, each joint/set of muscles were passively moved, and subjects attempted to voluntarily execute wrist/finger extension, elbow extension, and shoulder joint abduction.

\section{Data reduction}

Three median reaction times (total, premotor, and motor) were calculated from the rectified and smoothed EMG data, and force amplitude. Total reaction times were fractionated into two components as premotor (premovement*) reaction time (that is, central component defined as the time from stimulus onset until the EMG activity of the extensor muscles reached $30 \%$ of peak activity), and motor (movement*) reaction time (that is, peripheral component that started directly after premotor reaction time and ended with movement initiation as the peak force amplitude reached $30 \%){ }^{12324}$

For the sustained contraction task, the ability to maintain a maximum level of force was measured by root mean square error (RMSE, an overall measure of variability). Median RMSE was computed across the central portion five second interval of peak force amplitude.

Normality tests on the practice groups for each dependent measure were conducted before the data were analysed with separate mixed design analyses. These descriptive analyses revealed extreme scores in two tasks for three subjects, one in each group. Consistent with conventional statistical procedures these extreme scores were removed from all datasets. ${ }^{125-27}$ Subsequent analyses were conducted on 31 subjects, and normality was confirmed on the practice schedule groups across test sessions. All statistical tests were conducted with probability set at 0.05 , and Tukey-Kramer's procedure was used for multiple comparison follow up tests.

\section{RESULTS}

Box and block test

The number of blocks moved in the box and block test were analysed in a mixed design practice schedule (3: blocked,

${ }^{*}$ A reviewer mentioned that premovement time may be preferred to premotor reaction time and that movement time is preferred to motor reaction time. For consistency across studies, the classic usage of premotor and motor reaction times was maintained. random, and control) $\times$ test session (2: pretest and post-test) analysis of variance (ANOVA) with repeated measures on test session. The analysis indicated a significant two way interaction $(\mathrm{F}(2,28)=3.63, \mathrm{p}=0.039$; fig 1$)$. Follow up analysis showed that the motor capabilities of the three treatment groups were equivalent at the pretest. However, at the post-test, the number of blocks moved increased significantly for both the blocked and random practice groups in comparison with the control group. No reliable difference was found between the blocked and random practice groups.

\section{Total, premotor, and motor reaction time}

A third factor, impaired limb testing condition (unilateral or bilateral) was added as a second within-subjects factor. The practice schedule $\times$ test session $\times \operatorname{limb}(3 \times 2 \times 2)$ ANOVA indicated a significant test session main effect $(\mathrm{F}(\mathrm{l}, 28)=4.74, \mathrm{p}=0.038)$. Faster total reaction times were found on the post-test $($ median $=319 \mathrm{~ms}, \mathrm{SE}=11.18) v$ the pretest $($ median $=347 \mathrm{~ms}, \mathrm{SE}=13.68)$.

The premotor reaction time mixed design analysis revealed two significant effects: first, a test session main effect $(\mathrm{F}(\mathrm{l}, 28)=4.61, \mathrm{p}=0.04)$, and second, practice schedule $\times$ test session $\times$ limb interaction $(F(2,28)=4.56, p=0.019)$. Figure 2 shows the higher order three way interaction. Follow up comparisons indicated that the impaired limb in the unilateral and bilateral testing conditions showed faster premotor reaction times in the blocked practice group across the test sessions. In contrast, the random practice group only improved in the bilateral condition.

Analysis of motor reaction time indicated a significant limb main effect $(F(1,28)=9.26, p=0.005)$, as well as a reliable three way interaction $(\mathrm{F}(2,28)=3.64, \mathrm{p}=0.039$; fig 3$)$. Faster motor reaction times were identified for the blocked practice group during the post-test for both limb conditions. Further, the random practice group decreased median motor reaction times in the bilateral condition from the pretest to post-test, and no differences were found in the unilateral testing condition. In comparison with the control group during the bilateral situation, both practice groups showed faster motor reaction time improvements across the test sessions.

\section{Sustained muscle contraction}

The mixed design $3 \times 2 \times 2$ ANOVA on median RMSE during the sustained task indicated three reliable main effects: a practice schedule effect $(\mathrm{F}(2,28)=4.15, \mathrm{p}=0.026)$; a test

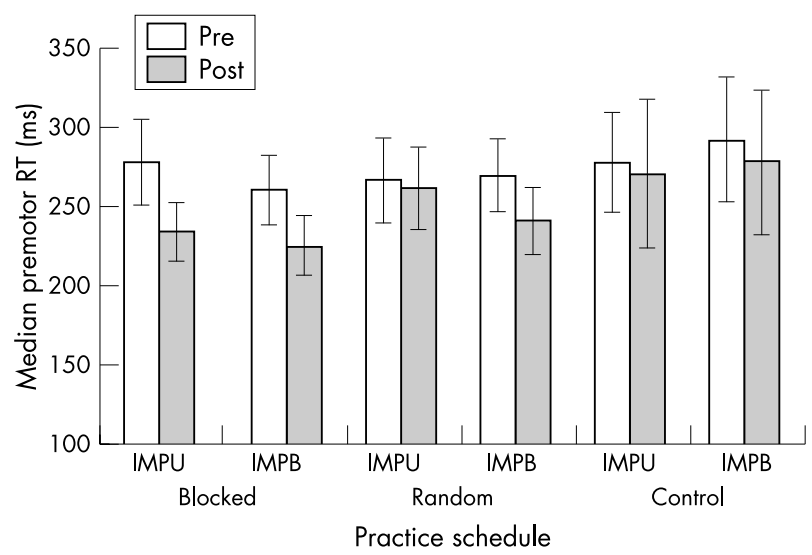

Figure 2 Median premotor reaction time and standard error bars for the tests sessions as a function of motor recovery practice schedules and limb conditions. IMPB, impaired limb in a bilateral testing condition; IMPU, impaired limb in a unilateral testing condition. 


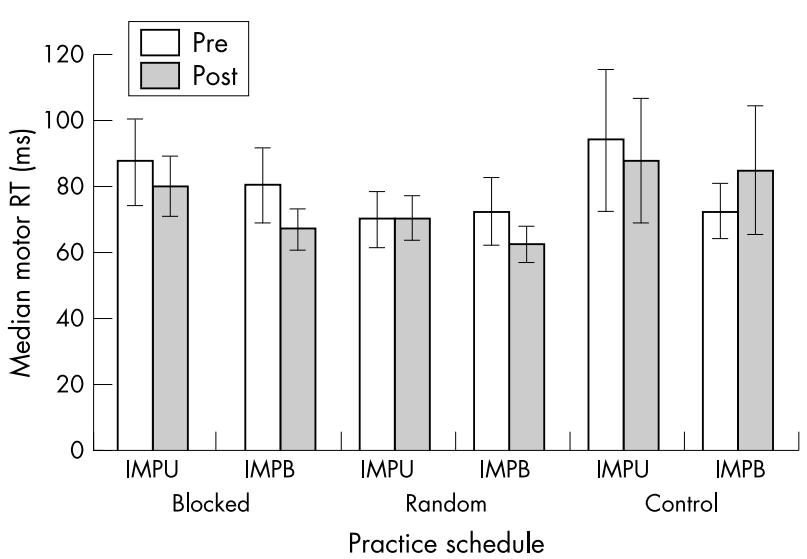

Figure 3 Median motor reaction time and standard error bars for the tests sessions as a function of motor recovery practice schedules and limb conditions. IMPB, impaired limb in a bilateral testing condition; IMPU, impaired limb in a unilateral testing condition.

session effect $(\mathrm{F}(1,28)=19.50, \mathrm{p}=0.0001)$; and a limb effect $(\mathrm{F}(\mathrm{l}, 28)=11.71, \mathrm{p}=0.001)$. In addition, two reliable interactions were found: practice schedule $\times$ test session $(\mathrm{F}(2,28)=7.67, \mathrm{p}=0.002)$, and practice schedule $\times$ limb $(\mathrm{F}(2,28)=8.43, \mathrm{p}=0.001)$. Follow up analysis on the practice schedule by test session interaction (fig 4 ) indicated equivalent practice groups at the pretest. Further, both the blocked and random groups decreased the amount of RMSE from the pretest to the post-test, and both groups were better than the control group at the post-test. No improvement was found for the control group across the test sessions.

Post hoc analysis on the practice schedule by limb interaction showed that in the bilateral testing condition the impaired limb of both the blocked and random practice groups showed less RMSE (blocked $=0.217$; random $=$ 0.258 ) than during the unilateral condition (blocked $=0.258$; random $=0.336$ ). In addition, both groups performed with less RMSE than the control group in both testing conditions ( unilateral $=0.375$; bilateral $=0.398$ ). These reliable median RMSE two way interactions are viewed as converging evidence favouring blocked and random practice.

\section{DISCUSSION}

The motor capabilities of chronic stroke patients were evaluated by behavioural measures before and after active neuromuscular stimulation was coupled with blocked and random practice. Analyses revealed distinct functional improvements for the blocked and random practice groups in comparison with the control group. Evidence favouring contextual interference was not as clean.

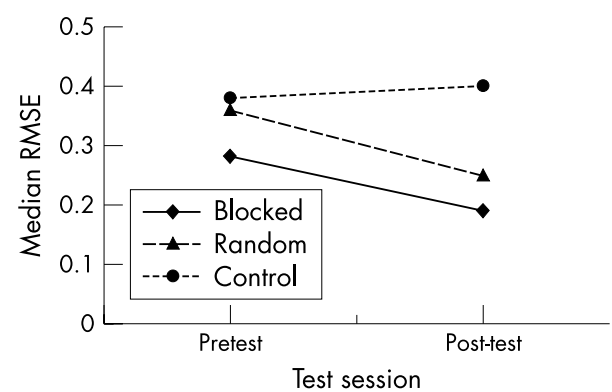

Figure 4 Median root mean square error (RMSE) for the motor recovery practice schedules as a function of test sessions.

\section{Contextual interference}

Two surprising findings are the equivalent performances of the blocked and random practice groups for the number of blocks moved and fractionated reaction time components. Based on motor learning studies, random practice was expected to excel in comparison to blocked practice..$^{5-8}$ However, the practice schedules coupled with active stimulation failed to differentiate the groups and consequently failed to support contextual interference. Even though the random practice group executed different purposeful movements on consecutive trials, the reported advantage for solving each motor problem multiple times during training was not found. One possible explanation concerns the supplemental active stimulation provided to a specific set of muscles when voluntary EMG activity reached threshold. Perhaps active neuromuscular stimulation attenuates the random practice motor learning advantage by increasing the capability of relearning upper extremity movements during blocked practice.

Further, Immink and Wright argued that practice schedules reflect low or high contextual interference depending on the amount of preparation time necessary before movement initiation. ${ }^{8}$ They state that in blocked practice appropriate movement parameters are loaded into working memory and maintained for an entire block of trials. ${ }^{8}$ On the contrary, in random practice the motor problem involved initiating and executing one of three different movements every trial, demanding processing time in working memory that is dissimilar to maintaining planned movement signals. ${ }^{8}$ However, the current findings suggest that the high contextual interference reported for random practice conditions may be attenuated with the addition of voluntarily initiated active neuromuscular stimulation.

\section{Sensorimotor integration theory}

Findings from each of the three measurement categories provide clear evidence supporting sensorimotor integration theory in that the active stimulation in conjunction with blocked and random practice shows progress toward recovery from chronic hemiparesis. First, increases in the number of blocks moved across the test sessions by the blocked and random practice groups showed decreased residual hemiparesis. Moreover, these improved motor capabilities are consistent with recent active stimulation motor recovery findings. Cauraugh et al reported improved motor capabilities in the upper extremity with active neuromuscular stimulation. ${ }^{9}$ Additional functional motor recovery improvements were found when EMG triggered stimulation was coupled with bilateral movements in the unimpaired limb.

Second, the premotor and motor fractionated reaction time results indicate an advantage for the blocked and random practice groups. Blocked and random practice decreased the processing time required for stimulus identification and response initiation. Essentially, the muscular activation patterns improved and thus voluntarily initiating movements in the impaired limb improved for both groups.

The shorter premotor and motor reaction times for the blocked and random practice groups are new findings and demonstrate progress in overcoming chronic hemiparesis. These findings extend the rapid muscle onset times to the practice environment. Further, the current results are consistent with classic fractionated reaction time findings in that premotor and motor reaction times were quicker to respond in the impaired limb after completing blocked and random practice/stimulation in the wrist/fingers, elbow, and shoulder joints. ${ }^{128}{ }^{29}$ The shorter times found in the bilateral situation for random practice are new findings that indicate an advantage for simultaneously initiating the same movement in both limbs. 
Further, support for sensorimotor integration theory was found in the decreased force variability during the sustained contraction task after rehabilitation. The blocked and random practice findings are similar to the force fluctuations reported by Enoka and colleagues-in old adults, force exerted during voluntary contraction fluctuates about a mean value. ${ }^{30}$ Across the current training sessions, the more consistent force fluctuations for the blocked and random practice groups support an explanation that force production results from the regularity that motor neurones discharge. ${ }^{30}$ Postintervention, the blocked and random subjects were able to minimise force fluctuations.

\section{Conclusions}

Cohen and Hallett elegantly chronicle the accumulating neural plasticity evidence concerning recovery of motor functions after stroke. ${ }^{31}$ The current unique active stimulation and practice schedule findings contribute to the evidence favouring neural plasticity of the motor cortex after stroke. However, the equivocal blocked and random practice results suggest that the paradox of contextual interference for motor skills does not apply to improved motor capabilities of chronic stroke impaired upper extremities (that is, six training hours). Rehabilitation specialist assisting patients with stroke induced chronic hemiparesis should not be concerned with implementing a random practice schedule for the current upper extremity movements. Blocked practice that follows a central to peripheral sequence is consistent with the notion that motor capabilities of stroke patients recover centrally before peripherally. ${ }^{32}$

\section{ACKNOWLEDGEMENTS}

An award to the first author from the American Heart Association, Florida/Puerto Rico Affiliate, supported this work.

\section{Authors' affiliations}

J H Cauraugh, S B Kim, University of Florida, Gainesville, Florida, USA

Competing interests: none declared

\section{REFERENCES}

1 Cauraugh JH, Kim SB. Two coupled motor recovery protocols are better than one: electromyogram-triggered neuromuscular stimulation and bilateral movements. Stroke 2002;33:1589-94.

2 Cramer SC, Bastings EP. Mapping clinically relevant plasticity after stroke. Neuropharmacology 2000:39:842-51.

3 Hallett M. Plasticity of the human motor cortex and recovery from stroke. Brain Res Rev 2001;36:169-74.

4 Hallett M. Recent advances in stroke rehabilitation. Neurorehabil Neural Repair 2002;16:211-17.

5 Schmidt RA, Bjork RA. New conceptualizations of practice: common principles in three paradigms suggest new concepts for training. Psychol Sci 1992;3:207-17.

6 Shumway-Cook A, Woollacott MH. Motor control: theory and practical applications, 2nd ed. Baltimore: Lippincott Williams and Wilkins, 2001.
7 Shea JB, Morgan RL. Contextual interference effects on the acquisition, retention, and transfer of a motor skill. J Exp Psychol: Hum Learn Mem 1979;5:179-87.

8 Immink MA, Wright DL. Motor programming during practice conditions high and low in contextual interference. J Exp Psychol: Hum Percept Perform $2001 ; 27: 423-37$.

9 Cauraugh JH, Light K, Kim SB, et al. Chronic motor dysfunction after stroke: recovering wrist and finger extension by electromyography triggered neuromuscular stimulation. Stroke 2000:31:1360-4.

10 Burridge $\mathbf{J H}$, Ladouceur M. Clinical and therapeutic applications of neuromuscular stimulation: A review of current use and speculation into future developments. Neuromodulation $2001 ; 4: 147-54$.

11 Cauraugh JH, Kim SB. Progress toward motor recovery with active neuromuscular stimulation: Muscle activation pattern evidence after a stroke. J Neurol Sci 2003;207:25-9.

12 Chae J, Yu D. Neuromuscular stimulation for motor relearning in hemiplegia. Crit Rev Phys Rehabil Med 1999;11:279-97.

13 Muellbacher W, Richards C, Ziemann U, et al. Improving hand function in chronic stroke. Arch Neurol 2002;59:1278-82.

14 Saper CB, Iversen S, Frackowiak R. Integration of sensory and motor function: the association areas of the cerebral cortex and the cognitive capabilities of the brain. In: Kandel ER, Schwartz JH, Jessell TM, eds. Principles of neural science, 4th ed. New York: McGraw-Hill, 2000:349-80.

15 Pleger B, Schwenkris P, Dinse H, et al. Pharmacological suppression of plastic changes in human primary somatosensory cortex after motor learning. Exp Brain Res 2002; Online publication: DOI 10.1007/s00221-022-1324-1.

16 Bernstein NA. The co-ordination and regulation of movements. Oxford: Pergamon Press, 1967.

17 Baratta RV, Solomonow M, Zhou BH, et al. Methods to reduce the variability of EMG power spectrum estimates. J Electromyogr Kinesiol 1998;8:279-85.

18 Macaluso A, De Vito G, Felici F, et al. Electromyogram changes during sustained contraction after resistance training in women in their 3rd and 8 th decades. Eur J Appl Physiol 2000;82:418-24.

19 Mathiowetz V, Vollard G, Kashman N, et al. Adult norms for the Box and Block test of manual dexterity. Am J Occup Ther 1985;39:386-91.

20 Ghez C, Krakauer J. The organization of movement. In: Kandel ER, Schwartz JH, Jessell TM, eds. Principles of neural science, 4th ed. New York: McGraw-Hill, 2000:653-73.

21 Krakauer J, Ghez C. Voluntary movement. In: Kandel ER, Schwartz JH, Jessell TM, eds. Principles of neural science, 4th ed. New York: McGraw-Hill, 2000:756-81.

22 Altman DG, Schulz KF. Concealing treatment allocation in randomized trials. BMJ 2001;323:446-7.

23 Weiss $A D$. The locus of reaction time change with set, motivation, and age. $J$ Gerontol 1965;20:60-4.

24 Botwinick J, Thompson LW. Premotor and motor components of reaction time. J Exp Psychol 1966;71:9-15.

25 Altman DG. Statistics in medical journals: some recent trends. Stat Med 2000;19:3275-89.

26 Winer BJ, Brown DR, Michels KM. Statistical principles in experimental design, 3rd ed. New York: McGraw-Hill, 1991.

27 Cauraugh JH. Experimental design and statistical decisions tutorial: comments on longitudinal ideomotor apraxia recovery. Neuropsychol Rehabil 2002;12:75-83.

28 Basmajian JV. Biofeedback principles and practice for clinicians, 3rd ed. Baltimore: Williams and Wilkins, 1989

29 Chae J, Yang G, Park B, et al. Delay in initiation and termination of muscle contraction, motor impairment, and physical disability in upper limb hemiparesis. Muscle Nerve 2002:25:568-75.

30 Christou EA, Shinohara M, Enoka RM. Fluctuations in acceleration during voluntary contractions lead to greater impairment of movement accuracy in old adults. J Appl Physiol 2003: online publication 10.1152.

31 Cohen LG, Hallett M. Neural plasticity and recovery of function. In: Greenwood RJ, Barnes MP, McMillan TM, et al, eds. Handbook of neurological rehabilitation, 2nd ed. Hove, England: Psychology Press, 2003:99-111.

32 Duncan PW, Lai SM. Stroke recovery. Top Stroke Rehabil 1997:4:51-8. 